1 Hacettepe Journal of Mathematics and Statistics

$\bigcap$ Volume 46 (6) (2017), 1053-1068

\title{
On the locally countable subalgebra of $C(X)$ whose local domain is cocountable
}

\author{
R. Mehri ${ }^{*}$ and R. Mohamadian ${ }^{\dagger}$
}

\begin{abstract}
In this paper, we present a new subring of $C(X)$ that contains the subring $C_{c}(X)$, the set of all continuous functions with countable image. Let $L_{c c}(X)=\left\{f \in C(X):\left|X \backslash C_{f}\right| \leq \aleph_{0}\right\}$, where $C_{f}$ is the union of all open subsets $U \subseteq X$ such that $|f(U)| \leq \aleph_{0}$. We observe that $L_{c c}(X)$ enjoys most of the important properties which are shared by $C(X)$ and $C_{c}(X)$. It is shown that any hereditary lindelöf scattered space is functionally countable. Spaces $X$ such that $L_{c c}(X)$ is regular (von Neumann) are characterized and it is shown that $\aleph_{0}$-selfinjectivity and regularity of $L_{c c}(X)$ coincide.
\end{abstract}

Keywords: functionally countable space, uncountable-open space, socle, locally $\mathfrak{c}$-scattered space, $\aleph_{0}$-selfinjective.

2000 AMS Classification: Primary 13A180, 54C40; Secondary 16A56.

Received: 04.05.2016 Accepted: 14.01.2017 Doi : 10.15672 /HJMS.2017.435

\section{Introduction}

Throughout this paper, the space $X$ stands for an uncountable completely regular Hausdorff space unless otherwise mentioned. We refer the reader to [9], [16], [32] and [1], [19] for undefined concepts and notations in this article relating to topology and algebra, respectively. The notation $C(X)$ and $C_{c}(X)$ are used for the ring of all real-valued continuous functions on $X$ and that of all continuous functions on $X$ with countable image, respectively. By $L_{c}(X)$ we mean the ring of all continuous functions that its local domains (note, if $f \in C(X)$, then its local domain, which is denoted by $C_{f}$, is defined by $C_{f}=\bigcup\left\{U \mid U\right.$ is open in $X$ and $\left.\left.|f(U)| \leqslant \aleph_{0}\right\}\right)$ are dense in $X$, see [23]. The subalgebra $C_{c}(X)$ of $C(X)$ is introduced and studied in [12] and [13]. Motivated by the fact that $C_{c}(X)$ is the largest subring of $C(X)$ whose elements have countable image, the subring $L_{c}(X)$ of $C(X)$ which lies between $C_{c}(X)$ and $C(X)$ is introduced in [23]. This subring

\footnotetext{
*Department of Mathematics, Shahid Chamran University of Ahvaz, Iran, Email: r-mehri@phdstu.scu.ac.ir

${ }^{\dagger}$ Department of Mathematics, Shahid Chamran University of Ahvaz, Iran, Email: mohamadian_r@scu.ac.ir
} 
naturally leads us to consider a new subring of $C(X)$, namely $L_{c c}(X)$, which lies between $C_{c}(X)$ and $L_{c}(X)$. Analogous to the main objective of research in context of $C(X)$, we will try to record some useful facts about $L_{c c}(X)$ and indicate the relations between topological properties of $X$ and the algebraic properties of $L_{c c}(X)$. In this paper we are interested in detecting topological spaces $X$ for which $L_{c c}(X)=L_{c}(X)$ and find spaces $X$ such that $L_{c c}(X)=C(X)$ and $L_{c c}(X)=C_{c}(X)$. In Section 3, we give some facts regarding the equality of $L_{c c}(X)$ with the rings $C(X), C_{c}(X)$ and $L_{c}(X)$. Also similar to a classical result due to Rudin in [30] and Pelczynski and Semadeni in [28], which says that a compact space $X$ is scattered (i.e., every nonempty subspace of $X$ contains an isolated point) if and only if $C(X)=C_{c}(X)$ (note, it is called RPS-Theorem, see [12]), it is shown that if $X$ is an uncountable-open locally compact space then $X$ is locally cscattered space if and only if $L_{c c}(X)=C(X)$, see Definitions 3.8 and 3.14. We introduce $z_{l c}$-ideals in Section 4 and observe that the sum of a collection of $z_{l c}$-ideals that is not the entire ring $L_{c c}(X)$ is $z_{l c}$-ideal. Also it is observed that the sum of a collection of minimal prime ideals in $L_{c c}(X)$ is a prime ideal in $L_{c c}(X)$ or the entire ring $L_{c c}(X)$. The topological spaces in which closed sets and points not belonging to them are separated by elements of $L_{c c}(X)$, are called co-locally countable completely regular space (briefly, lcccompletely regular) and are introduced in Section 5. In this section, spaces $X$ for which $L_{c c}(X)$ is regular are characterized both topologically and algebraically. Also similarly to the rings $C(X), C_{c}(X)$ and $L_{c}(X)$, we show that $L_{c c}(X)$ is a regular ring if and only if it is $\aleph_{0}$-selfinjective. The socle of $C(X)$ (i.e., $\left.C_{F}(X)\right)$ which is in fact a direct sum of minimal ideals of $C(X)$ is topologically characterized in [24]. The socle of $C_{c}(X)$, denoted by $\operatorname{Soc}\left(C_{c}(X)\right)$, is studied and also topologically characterized in [13, Proposition 5.3], and spaces $X$ for which $\operatorname{Soc}\left(C_{c}(X)\right)=C_{F}(X)$ are determined in [13, Theorem 5.6], too. In Section 6, similarly to the ring $L_{c}(X)$ in [23], we characterize the socle of $L_{c c}(X)$ both topologically and algebraically. Also, spaces $X$ for which $\operatorname{Soc}\left(L_{c c}(X)\right)=\operatorname{Soc}\left(C_{c}(X)\right)$ and $\operatorname{Soc}\left(L_{c c}(X)\right)=C_{F}(X)$ are characterized.

\section{Notation and preliminaries}

In this section for the sake of the reader in details, we recall the definitions and the notations used in this article. We also mention some background results from [12] and [23]. It is well-known that the semiprime ideals of a commutative ring are precisely the intersections of prime ideals, see [16,2B(3)]. An ideal $I$ of a commutative ring $R$ is called pseudoprime if $a, b \in R$ and $a b=0$ implies $a \in I$ or $b \in I$. Recall that for $f \in C(X), Z(f)$ denotes its zero-set, i.e., $Z(f)=\{x \in X: f(x)=0\}$ and the set-theoretic complement of this zero-set is known as a cozero-set and we denote it by cozf. The Stone-Čech compactification of $X$ is denoted by $\beta X$, and by $|X|$ we mean the cardinality of $X$. In this paper, $\omega_{1}$ and $\mathfrak{c}$ stand for the smallest uncountable ordinal and the power of continuum, respectively. Let $X$ and $Y$ be disjoint topological spaces, the free union of $X$ and $Y$ is denoted by $X \dot{\cup} Y$, see [32]. A space $X$ is called functionally countable if each member of $C(X)$ has countable image (i.e., $\left.C(X)=C_{c}(X)\right)$. An ideal $I$ of $C(X)$ (resp., $\left.C_{c}(X)\right)$ is said to be $z$-ideal (resp., $z_{c}$-ideal) of $C(X)$ (resp., $\left.C_{c}(X)\right)$ if $Z(f) \subseteq Z(g)$ where $f \in I$ and $g \in C(X)$ (resp., $\left.g \in C_{c}(X)\right)$ implies that $g \in I$. We recall that minimal prime ideals in $C(X)$ (resp., $\left.C_{c}(X)\right)$ are $z$-ideals (resp., $z_{c}$-ideals), see [16, Theorem 14.7] and [12, Corollary 3.4]. The socle of $C(X)$, denoted by $C_{F}(X)$, is the sum of all minimal ideals of $C(X)$, which is the intersection of all essential ideals in $C(X)$ (note, an ideal is essential if it intersects every nonzero ideal nontrivially). The socles of $C_{c}(X)$ and $L_{c}(X)$ are also characterized topologically, see [13], [23]. The following proposition which characterizes the socle of $C(X)$ topologically, is proved in [24]. 
2.1. Proposition. The socle $C_{F}(X)$ of $C(X)$ is a $z$-ideal consisting of all functions that vanish everywhere except on a finite subset of $X$.

2.2. Definition. Let $f \in C(X)$ and $C_{f}$ be the union of all open sets $U \subseteq X$ such that $f(U)$ is countable, i.e.,

$$
C_{f}=\bigcup\left\{U \mid U \text { is open in } X \text { and }|f(U)| \leqslant \aleph_{0}\right\} .
$$

We call $C_{f}$ the local domain of $f$ and denote by $L_{c}(X)$ the set of all $f \in C(X)$ such that $C_{f}$ is dense in $X$.

We cite the following fact which is in [23].

2.3. Lemma. For the space $X$ the following statements hold.

(1) If $f, g \in C(X)$, then $C_{f+g} \supseteq C_{f} \cap C_{g}$.

(2) If $f, g \in C(X)$, then $C_{f g} \supseteq C_{f} \cap C_{g}$.

(3) If $f \in C(X)$, then $C_{|f|}=C_{f}$.

(4) If $f \in C(X)$, then $C_{\frac{1}{f}}=C_{f}$.

\section{The subalgebra $L_{c c}(X)$ of $C(X)$}

3.1. Definition. We define $L_{c c}(X)$ to be the set of all $f \in C(X)$ whose local domain is cocountable, i.e.,

$$
L_{c c}(X)=\left\{f \in C(X):\left|X \backslash C_{f}\right| \leqslant \aleph_{0}\right\} .
$$

It is obvious that $L_{c c}(X)$ is a subring of $C(X)$ containing $C_{c}(X)$. In fact $L_{c c}(X)$ is a subalgebra as well as a sublattice of $C(X)$ and we call it the co-locally functionally countable subalgebra of $C(X)$.

3.2. Remark. Let $X$ be an arbitrary space, then the relation $L_{c c}(X) \subseteq L_{c}(X)$ holds, for if $f \in L_{c c}(X)$ and $U$ is an open subset in $X$, then $U \subseteq C_{f}$ in case $\bar{U}$ is countable, and if $U$ is uncountable, $U$ meets $C_{f}$ (note, $C_{f}$ is cocountable), hence $C_{f}$ is dense in $X$.

For any space $X$ the relation $C_{F}(X) \subseteq C^{F}(X) \subseteq C_{c}(X) \subseteq L_{c c}(X) \subseteq L_{c}(X) \subseteq C(X)$ holds, where $C^{F}(X)=\{f \in C(X):|f(X)|<\infty\}$ and $C_{F}(X)$ is the socle of $C(\bar{X})$. The following example shows that the equality between any two of these objects may not necessarily hold.

3.3. Example. Let $A=(-\infty, 0)$. Suppose that the basic neighborhood of $x \in A$ when $x \in[-1,0) \cup\{x \in \mathbb{Q}: x<-1\}$ is $\{x\}$ and for the other points of $A$, the basic neighborhood be the usual open intervals containing $x$. Moreover, let $B=[0, \infty)$ and the basic neighborhood of $x$ when $x \in\{x \in \mathbb{Q}: 0 \leqslant x \leqslant 1\}$ is $\{x\}$ and for the rest of the members, $y$ say, of $B$ the basic neighborhood be the usual open intervals containing $y$. Let $X=A \cup \dot{\cup} B$, be the free union of $A$ and $B$. The function $f: X \rightarrow \mathbb{R}$, where $f(x)=x$ is a continuous function and $f \notin L_{c}(X)$. The function $g_{B}: B \rightarrow \mathbb{R}$, such that $g_{B}(x)=0$ for $x>1$ and $g_{B}(x)=x$ otherwise, is a continuous function on $B$. Now we define $g: X \rightarrow \mathbb{R}$, where $g(x)=g_{B}(x)$ if $x \in B$ and $g(x)=0$ for $x \in A$. The function $g$ is continuous with $C_{g}=\mathbb{R} \backslash\left((0,1) \cap \mathbb{Q}^{c}\right)$, hence $g \in L_{c}(X) \backslash L_{c c}(X)$. The function $h_{A}: A \rightarrow \mathbb{R}$, where $h_{A}(x)=x$ for $x \in[-1,0)$ and $h_{A}(x)=0$ otherwise, is a continuous function on $A$. The function $h: X \rightarrow \mathbb{R}$, such that $h(x)=h_{A}(x)$ for $x \in A$ and $h(x)=0$ for $x \in B$ is continuous function on $X$ with $C_{h}=\mathbb{R}$ and we have $h \in L_{c c}(X) \backslash C_{c}(X)$. Also one can easily see that the set $D=\left\{\frac{1}{n}: n \in \mathbb{N}\right\} \cup\{0\}$ is a clopen subset of $B$ and hence it is clopen in $X$. The function $t: X \rightarrow \mathbb{R}$, where $t(x)=x$ for $x \in D$ and $t(x)=0$ otherwise, is continuous and $t \in C_{c}(X) \backslash C^{F}(X)$. Finally the function $s: X \rightarrow \mathbb{R}$, which equals to 1 on $A$ and vanishes on $B$ is a continuous function and $s \in C^{F}(X) \backslash C_{F}(X)$. 
The proof of the following lemma is elementary and we state it without proof.

3.4. Lemma. For the space $X$ the following statements hold.

(1) If $f, g \in L_{c c}(X)$, then $f+g \in L_{c c}(X)$ and $f g \in L_{c c}(X)$.

(2) If $f \in L_{c c}(X)$, then $|f| \in L_{c c}(X)$.

(3) Let $f$ be a unit element in $C(X)$, then $f \in L_{c c}(X)$ if and only if $\frac{1}{f} \in L_{c c}(X)$.

3.5. Corollary. $L_{c c}(X)$ is a sublattice of $C(X)$.

3.6. Definition. Let $f \in C(X)$ and $C_{f}^{F}$ be the union of all open sets $U \subseteq X$ such that $f(U)$ is finite, i.e.,

$$
C_{f}^{F}=\bigcup\{U \mid U \text { is open in } X \text { and }|f(U)|<\infty\} .
$$

Denote by $L_{c F}(X)$ the set of all $f \in C(X)$ such that $C_{f}^{F}$ is cocountable, and call it co-locally functionally finite subalgebra of $C(X)$, i.e.,

$$
L_{c F}(X)=\left\{f \in C(X):\left|X \backslash C_{f}^{F}\right| \leqslant \aleph_{0}\right\} .
$$

In a special case, for $f \in C(X)$ let $C_{f}^{c}$ be the union of all open sets $U \subseteq X$ such that $f(U)$ is constant, i.e.,

$$
C_{f}^{c}=\bigcup\{U \mid U \text { is open in } X \text { and }|f(U)|=1\} .
$$

We define $L_{c 1}(X)$ to be the set of all $f \in C(X)$ such that $C_{f}^{c}$ be cocountable in $X$, and call it co-locally functionally constant subalgebra of $C(X)$, i.e.,

$$
L_{c 1}(X)=\left\{f \in C(X):\left|X \backslash C_{f}^{c}\right| \leqslant \aleph_{0}\right\} .
$$

Clearly, $L_{c F}(X)$ and $L_{c 1}(X)$ are subalgebra of $L_{c c}(X)$.

3.7. Remark. We note that the previous lemma and corollary are also valid for $L_{c F}(X)$ and $L_{c 1}(X)$.

We are interested in characterizing topological spaces $X$ for which $L_{c c}(X)=C(X)$ and also the spaces $X$ such that $L_{c c}(X)=L_{c}(X)$. Recall that a point $x \in X$ is called countably isolated if there exists a countable open set in $X$ containing $x$, also a space $X$ is called countably discrete if all of its points are countably isolated, see [15]. If $X$ is a countably discrete space, then $L_{c c}(X)=C(X)$, for if $f \in C(X)$ and $x \in X$ then there exists an open set $U_{x}$ in $X$ that contains $x$ and $\left|U_{x}\right| \leqslant \aleph_{0}$. Therefore $C_{f}=X$ and $f \in L_{c c}(X)$. In fact we have $L_{c 1}(X)=L_{c F}(X)=L_{c c}(X)=C(X)$.

3.8. Definition. A space $X$ is called uncountable-open if any uncountable subset of $X$ has nonempty interior.

Clearly, discrete spaces are uncountable-open spaces. Additionally there are many non-discrete spaces that are uncountable-open, for instance, one point compactification of an uncountable discrete space is one of them. Also if $X$ is an uncountable discrete space and $Y$ is an arbitrary countable space then $X \dot{\cup} Y$, the free union of them, is uncountable-open space. The next proposition is a fact in this class of spaces.

3.9. Proposition. For a space $X$ the following statements are equivalent.

(1) $X$ is an uncountable-open space.

(2) The complement of any dense subset of $X$ is at most countable.

(3) For any subset $A$ of $X, A \backslash$ int $A$ is countable.

(4) For any subset $A$ of $X, \operatorname{cl} A \backslash A$ is countable.

Proof. $(1 \Rightarrow 2)$ If $A$ is a dense subset of $X$ then $X \backslash A$ has empty interior and hence by our assumption, $X \backslash A$ must be countable.

$(2 \Rightarrow 3)$ For any subset $A$ of $X$, the set $A \backslash$ int $A$ has empty interior and hence its 
complement is dense in $X$.

$(3 \Rightarrow 4)$ For any subset $A$ of $X$ the relation $\operatorname{cl} A \backslash A=(X \backslash A) \backslash \operatorname{int}(X \backslash A)$ holds, therefore $\operatorname{cl} A \backslash A$ is countable.

$(4 \Rightarrow 1)$ Let $A$ be an uncountable subset of $X$. If int $A=\emptyset$ then $X \backslash A$ is a dense subset of $X$ and hence $A=\operatorname{cl}(X \backslash A) \backslash(X \backslash A)$ is countable, a contradiction.

In view of the previous proposition, the proof of the next lemma is evident.

3.10. Lemma. If $X$ is an uncountable-open space, then $L_{c c}(X)=L_{c}(X)$.

3.11. Remark. The converse of the above lemma is not true in general. The space of all ordinals less than the smallest uncountable ordinal $\omega_{1}$ (i.e., $W=W\left(\omega_{1}\right)$ ), is not uncountable-open space. In fact, the set of all limit ordinals in $W$ is an uncountable set with empty interior. Since any function $f \in C(W)$ is constant on a tail of $W$, we infer that $L_{c c}(W)=L_{c}(W)$, see $[16,5.12]$.

Let us recall some facts about scattered spaces. A space $X$ is called scattered if any nonempty subset of $X$ has an isolated point with respect to its induced topology. In fact the space $X$ is scattered if and only if any nonempty closed subset of $X$ has an isolated point. For a cardinal number $\alpha$, a space $X$ is called $\alpha$-scattered, if any subset $A$ of $X$ with $|A| \geq \alpha$ has an isolated point. The next lemma present a class of spaces $X$ that $L_{c c}(X)$ and $C(X)$ coincide.

3.12. Lemma. If $X$ is an uncountable-open and $\mathfrak{c}$-scattered space, then $L_{c c}(X)=C(X)$.

Proof. Let $f \in C(X)$ and $X \backslash C_{f}$ be an uncountable subset of $X$. Since $X$ is uncountableopen space, we infer that there exists an uncountable open set $U$ in $X$ such that $U \subseteq$ $X \backslash C_{f}$ (note, otherwise $U \subseteq C_{f}$ ). Since $X$ is c-scattered space, we infer that $U$ has an isolated point with respect to the induced topology on $U, x$ say, and hence $x$ is an isolated point in $X$, too. Consequently, $x \in C_{f}$, which is the desired contradiction.

3.13. Corollary. For an uncountable-open scattered space $X$ we have $L_{c c}(X)=C(X)$.

3.14. Definition. A space $X$ is called locally c-scattered if given any $f \in C(X)$ and an uncountable open set $U$, there exists a compact subset $V_{f}$, with $\emptyset \neq V_{f}^{\circ} \subseteq U$ and $\left|f\left(V_{f}^{\circ}\right)\right| \leq \aleph_{0}$.

Similarly, a space $X$ is called locally scattered space if given any $f \in C(X)$ and a nonempty open set $U$, there exists a compact subset $V_{f}$, with $\emptyset \neq V_{f}^{\circ} \subseteq U$ and $\left|f\left(V_{f}^{\circ}\right)\right| \leq \aleph_{0}$, see [23, Definition 2.14]. Clearly, every locally scattered space is a locally $\mathfrak{c}$-scattered space and the space $\beta X$ (i.e., the Stone-Čech compactification of $X$ ) is locally scattered, where $X$ is a discrete space. Also every $\mathfrak{c}$-scattered space is locally c-scattered, for if $X$ is a c-scattered space, $f \in C(X)$ and $U$ be an open set in $X$ with $|U| \geq \mathfrak{c}$, then $U$ has an isolated point, $\{x\}$ say, therefore $\{x\}$ is the desired compact subset of $X$. The converse of this fact is not true, for example, $\beta \mathbb{N}$ is a locally $\mathfrak{c}$-scattered space which is not $\mathfrak{c}$-scattered, for $\beta \mathbb{N} \backslash \mathbb{N}$ has no isolated point. The following example shows that a locally $\mathfrak{c}$-scattered space need not be locally scattered space.

3.15. Example. Assume that $\mathbb{Q}^{c}$, the set of irrational numbers, equipped with discrete topology and $\mathbb{Q}$, the set of rational numbers, be the subspace of $\mathbb{R}$ with the usual topology. The space $X=\mathbb{Q} \cup \mathbb{Q}^{c}$, the free union of $\mathbb{Q}$ and $\mathbb{Q}^{c}$, is locally $\mathfrak{c}$-scattered space. The function $f: X \rightarrow \mathbb{R}$, where $f(x)=x$ is a continuous function on $X$ and $\mathbb{Q}$ is an open subset in $X$, but there is no compact neighborhood subset in $\mathbb{Q}$. Thus $X$ is not locally scattered space.

3.16. Lemma. Any open $C$-embedded subset of a locally $\mathfrak{c}$-scattered space is locally $\mathfrak{c}$ scattered. 
Proof. Let $Y$ be an open $C$-embedded subset of a locally c-scattered space $X$. Let $U$ be an uncountable open set in $Y$ and $f \in C(Y)$. Since $Y$ is $C$-embedded, we infer that there exists $g \in C(X)$ such that $\left.g\right|_{Y}=f$. Clearly, $U$ is an open subset of $X$ and by our assumption, there exists a compact subset $V_{g}$ in $U$ with $\emptyset \neq V_{g}^{\circ} \subseteq U \subseteq Y$ and $\left|g\left(V_{g}^{\circ}\right)\right| \leq \aleph_{0}$. Therefore $V_{g}$ is a compact subset of $Y$ with $\left|f\left(V_{g}^{\circ}\right)\right|=\left|g\left(V_{g}^{\circ}\right)\right| \leq \aleph_{0}$, i.e., $Y$ is locally c-scattered.

We recall that a Hausdorff space $X$ is locally compact if and only if each point in $X$ has a compact neighborhood. Clearly, every compact Hausdorff space is locally compact. The following result is in order.

3.17. Theorem. Let $X$ be a locally compact space. If $L_{c c}(X)=C(X)$ then $X$ is locally $\mathfrak{c}$-scattered space. Moreover, if $X$ is uncountable-open space then the converse holds.

Proof. First, we assume that $X$ is a locally compact space and $L_{c c}(X)=C(X)$. Let $f \in C(X)$ and $U$ be an open set of $X$ with $|U| \geqslant \mathfrak{c}$. Since $\left|X \backslash C_{f}\right| \leqslant \aleph_{0}$, we infer that $U$ meets $C_{f}$ and hence there exists an open set $V \subseteq X$ such that $|f(V)| \leq \aleph_{0}$ and $V \cap U \neq \emptyset$. As $X$ is locally compact space, $V \cap U$ is locally compact, too. Thus any neighborhood of a point $x \in V \cap U$ contains a compact neighborhood, $V_{f}$ say, of $x$. Clearly, $V_{f}^{\circ} \subseteq U$ and $\left|f\left(V_{f}^{\circ}\right)\right| \leq \aleph_{0}$. Finally, suppose that $L_{c c}(X) \neq C(X)$ and get a contradiction. If $f \in C(X) \backslash L_{c c}(X)$, then since $X$ is an uncountable-open space, we infer that there exists an open set $U$ with $U \subseteq X \backslash C_{f}$ and a compact neighborhood $V_{f}$ such that $V_{f}^{\circ} \subseteq U$ and $\left|f\left(V_{f}^{\circ}\right)\right| \leq \aleph_{0}$. Therefore $U \subseteq X \backslash C_{f} \subseteq X \backslash V_{f}^{\circ}$, which is the desired contradiction.

Let us recall that a topological space $X$ is said to be Lindelöf space if every open cover of $X$ has a countable subcover. A Lindelöf space $X$ is called hereditary if every subspace of $X$ is Lindelöf. Clearly, every second-countable space is hereditary Lindelöf [32, 16E1]. A subset $Y$ of a space $X$ is called preopen, if $Y \subseteq \operatorname{int}(c l Y)$, also a space $X$ is called strongly Lindelöf if every preopen cover of $X$ admits a countable subcover, see [11].

3.18. Proposition. The following statements hold.

(1) If $X$ is hereditary Lindelöf space, then $L_{c c}(X)=C_{c}(X)$.

(2) If $X$ is strongly Lindelöf space, then $L_{c c}(X)=L_{c}(X)$.

(3) If $X$ is hereditary Lindelöf, c-scattered space then $L_{c c}(X)=C(X)$.

Proof. (1) Let $f \in L_{c c}(X)$ and $A=\left\{U_{i}: i \in I\right\}$ be the set of all open sets in $X$, where each $f\left(U_{i}\right), i \in I$ has countable image. Since $X$ is hereditary Lindelöf space, we infer that $A$ has a countable subfamily, $\left\{U_{i_{n}}: n \in \mathbb{N}\right\}$, that covers $C_{f}$. Therefore $\left|f\left(C_{f}\right)\right|=\left|f\left(\bigcup_{n \in \mathbb{N}} U_{i_{n}}\right)\right|=\left|\bigcup_{n \in \mathbb{N}} f\left(U_{i_{n}}\right)\right| \leqslant \aleph_{0}$, so $f \in C_{c}(X)$.

(2) If $f \in L_{c}(X)$, then the collection $\mathcal{A}=\left\{C_{f} \cup\{x\}: x \in X \backslash C_{f}\right\}$ is a preopen cover of $X$. By our assumption, $\mathcal{A}$ has a countable subcover, i.e., $X=\bigcup_{n \in \mathbb{N}}\left(C_{f} \cup\left\{x_{n}\right\}\right)$. Therefore $C_{f}$ is cocountable and $f \in L_{c c}(X)$.

(3) Suppose that $f \in C(X)$ with $\left|X \backslash C_{f}\right| \geqslant \mathfrak{c}$ and seek a contradiction. Since $X$ is c-scattered space, we infer that $X \backslash C_{f}$ has an isolated point, $\left\{x_{0}\right\}$ say, therefore there exists an open set $U \subseteq X$ such that $U \cap\left(X \backslash C_{f}\right)=\left\{x_{0}\right\}$. The set $U \backslash\left\{x_{0}\right\}$ is an open set in $X$ and $U \backslash\left\{x_{0}\right\} \subseteq C_{f}$. Since $X$ is hereditary Lindelöf, we infer that $C_{f}=\bigcup_{n \in \mathbb{N}} U_{n}$, where $\left|f\left(U_{n}\right)\right| \leq \aleph_{0}$, for all $n \in \mathbb{N}$. Therefore $\left|f\left(C_{f}\right)\right| \leq \aleph_{0}$ and we have $\left|f\left(U \backslash\left\{x_{0}\right\}\right)\right| \leq \aleph_{0}$ which implies $f(U)$ is countable, a contradiction.

3.19. Corollary. Every hereditary Lindelöf and scattered space is functionally countable.

We recall that if the set of open neighborhoods of a point $p$ in $X$ is closed under countable intersection, then $p$ is called a $P$-point. The set of all $P$-points of $X$ is denoted by $\mathcal{P}_{X}$ and $X$ is called a $P$-space if $\mathcal{P}_{X}=X$. The next immediate result implies that for a $P$-space $X$, the rings $L_{c c}(X)$ and $C(X)$ coincide. 
3.20. Proposition. If $X$ is a $P$-space, then $L_{c c}(X)=C(X)$.

Proof. Let $f \in C(X)$ and $x$ be an arbitrary point of $X$. Since $X$ is a $P$-space, there exists an open neighborhood $U_{x}$ of $x$ such that $f$ is constant on $U_{x}$, see[16, 4L(3)]. Therefore $C_{f}=X$ and hence $f \in L_{c c}(X)$.

3.21. Remark. It is worth mentioning that the converse of the previous proposition is not true in general, for example, the space of all ordinals less than the smallest uncountable ordinal $\omega_{1}$ (i.e., $\left.W=W\left(\omega_{1}\right)\right)$ is not a $P$-space, but $L_{c c}(W)=C(W)$. Also by [16, $13 \mathrm{P}]$ there exists a $P$-space $X$ without isolated points with $L_{c c}(X)=C(X)$.

Let us recall that a topological space $X$ is called locally functionally countable if every point $x \in X$ is countably $P$-point, in the sense that there exists an open neighborhood $U_{x}$ of $x$ such that $C\left(U_{x}\right)=C_{c}\left(U_{x}\right)$, see [18] and [23]. The following result shows that for every locally functionally countable space $X$, the spaces $C(X)$ and $L_{c c}(X)$ coincide.

3.22. Proposition. If $X$ is a locally functionally countable space, then $L_{c c}(X)=C(X)$.

Proof. Let $f \in C(X)$ and $x \in X$. Since $X$ is locally functionally countable space, we infer that there exists an open neighborhood $U_{x}$ of $x$ such that $C\left(U_{x}\right)=C_{c}\left(U_{x}\right)$. Obviously, $\left|f\left(U_{x}\right)\right|=\left|\left(\left.f\right|_{U_{x}}\right)\left(U_{x}\right)\right| \leq \aleph_{0}$. Hence $U_{x} \subseteq C_{f}$ and $C_{f}=X$, i.e., $L_{c c}(X)=C(X)$.

We note that the converse of the previous result is not true in general, in Example 4.7 for the space $X, C(X)=L_{c c}(X)$, but it is not locally functionally countable (note, no rational number in $X$ is a countably $P$-point).

\section{4. $z_{l c}$-ideals}

In this section we state some facts about $L_{c c}(X)$. We remind the reader that similarly to $C_{c}(X)$ and $L_{c}(X)$, many results in the context of $C(X)$ can be proved for $L_{c c}(X)$ in natural way, see [12] and [23]. Similarly to the positive element of the rings $C(X)$ and $L_{c}(X)$, if $f \in L_{c c}(X)$ and $f>0$, then there exists $g \in L_{c c}(X)$ with $f=g^{2}$. We also remark that if $f \in L_{c c}(X)$, then $f^{r} \in L_{c c}(X)$ for any $r \in \mathbb{R}$ that $f^{r}$ is defined. It is obvious that similar to the ring $C(X)$, all positive units in $L_{c c}(X)$ have the same number of square roots, see $[16,1 \mathrm{~B}(1)]$. The following proposition is the counterpart of $[16,1 \mathrm{D}(1)]$ and $\left[12\right.$, Lemma 2.4] for $L_{c c}(X)$.

4.1. Proposition. If $f, g \in L_{c c}(X)$ and $Z(g) \subseteq$ int $Z(f)$, then $f$ is a multiple of $g$.

Proof. Put

$$
h(x)=\left\{\begin{aligned}
0 & , x \in Z(f) \\
\frac{f(x)}{g(x)} & , x \in X \backslash \operatorname{int} Z(f)
\end{aligned}\right.
$$

clearly, $h \in C(X)$ and $C_{h} \supseteq C_{g} \cap C_{f}$, so $\left|X \backslash C_{h}\right| \leqslant \aleph_{0}$. Hence $h \in L_{c c}(X)$ and $f=h g$.

4.2. Corollary. If $f, g \in L_{c c}(X)$ and $|f| \leqslant|g|^{r}, r>1$, then $f=h g$ for some $h \in$ $L_{c c}(X)$. In particular, if $|f| \leqslant|g|$ then whenever $f^{r}$ is defined for $r>1, f^{r}$ is a multiple of $g$.

Proof. Put

$$
h(x)=\left\{\begin{aligned}
0 & , x \in Z(g) \\
\frac{f(x)}{g(x)} & , x \notin Z(g)
\end{aligned}\right.
$$

thus $h \in C(X)$ and $C_{h} \supseteq C_{g} \cap C_{f}$. Hence $h \in L_{c c}(X)$ and $f=h g$. 
4.3. Corollary. If $f \in L_{c c}(X)$, then there exists a positive unit $u \in L_{c c}(X)$ with $(-1 \vee$ $f) \wedge 1=u f$. Therefore the functions $f$ and $(-1 \vee f) \wedge 1$ belong to exactly the same ideals in $L_{c c}(X)$.

Proof. Let

$$
u(x)=\left\{\begin{aligned}
1 & ,|f(x)| \leqslant 1 \\
\frac{1}{|f(x)|} & ,|f(x)| \geqslant 1
\end{aligned}\right.
$$

obviously, $u \in C(X)$ and $C_{f}=C_{u}$ (i.e., $\left.u \in L_{c c}(X)\right)$ with $(-1 \vee f) \wedge 1=u f$.

Convention. For a topological space $X$, we denote by $Z_{l c}(X)$ the set of all zero-sets of $L_{c c}(X)$.

4.4. Definition. Two subsets $A$ and $B$ of a topological space $X$ are said to be co-locally countably separated (briefly, lcc-separated) in $X$ if there exists an element $f \in L_{c c}(X)$ such that $f(A)=1, f(B)=0$.

The proof of the following theorem which is similar to [12, Theorem 2.8] and [23, Theorem 3.10], is omitted.

4.5. Theorem. Two sets are lcc-separated if and only if they are contained in disjoint members of $Z_{l c}(X)$. Moreover, lcc-separated sets have disjoint zero-set neighborhoods in $Z_{l c}(X)$.

4.6. Definition. A nonempty subfamily $\mathcal{F}$ of $Z_{l c}(X)$ is called a $z_{l c}$-filter on $X$ if $\mathcal{F}$ satisfies the following conditions.

(1) $\emptyset \notin \mathcal{F}$.

(2) If $Z_{1}, Z_{2} \in \mathcal{F}$, then $Z_{1} \cap Z_{2} \in \mathcal{F}$.

(3) If $Z_{1} \in \mathcal{F}$ and $Z_{2} \in Z_{l c}(X)$ such that $Z_{1} \subseteq Z_{2}$ then $Z_{2} \in \mathcal{F}$.

By a $z_{l c}$-ultrafilter on $X$, it is meant a maximal $z_{l c}$-filter, i.e., one that is not contained in any other $z_{l c}$-filter. A $z_{l c}$-filter $\mathcal{F}$ is called prime $z_{l c}$-filter if $Z_{1} \cup Z_{2} \in \mathcal{F}$, where $Z_{1}, Z_{2} \in Z_{l c}(X)$, then $Z_{1} \in \mathcal{F}$ or $Z_{2} \in \mathcal{F}$. Clearly every $z_{l c}$-ultrafilter is prime $z_{l c}$-filter. If $I$ is an ideal of $L_{c c}(X)$, then $Z_{l c}[I]=\{Z(f): f \in I\}$ is a $z_{l c}$-filter on $X$. Conversely, if $\mathcal{F}$ is a $z_{l c}$-filter on $X$, then $Z^{-1}[\mathcal{F}]=\left\{f \in L_{c c}(X): Z(f) \in \mathcal{F}\right\}$ is an ideal of $L_{c c}(X)$. Moreover, every $z_{l c}$-filter $\mathcal{F}$ is of the form $\mathcal{F}=Z_{l c}[I]$ for some ideal $I$ of $L_{c c}(X)$ and for an ideal $J$ of $L_{c c}(X), Z^{-1} Z_{l c}[J]$ is an ideal of $L_{c c}(X)$ containing $J$. The following example shows that the latter inclusion may be proper.

4.7. Example. Let for any irrational number, say $x$, the basic neighborhood be $\{x\}$ and for the rest of the real numbers, i.e. $x \in \mathbb{Q}$, the basic neighborhood be the usual open intervals containing $x$. For this topology on $X=\mathbb{R}$ we have $L_{c c}(X)=C(X)$ and therefore $i$ belongs to $L_{c c}(X)$, where $i$ is the identity function. For the ideal $I=(i)$ of $L_{c c}(X)$ the relation $Z_{l c}[I]=\{0\}$ holds. Now, it is clear that the function $f(x)=x^{\frac{1}{3}}$ belongs to $L_{c c}(X)$ but $f \in Z^{-1} Z_{l c}[I] \backslash I$.

4.8. Definition. An ideal I of $L_{c c}(X)$ is called $z_{l c^{-}}$ideal if whenever $Z(f) \in Z_{l c}[I]$ and $f \in L_{c c}(X)$, then $f \in I$.

Obviously, every $z_{l c^{-}}$ideal is an intersection of prime ideals of $L_{c c}(X)$. Again we recall that the proofs of the following facts are the same as the proofs of their counterparts in $C(X), C_{c}(X)$ and $L_{c}(X)$, see [12], [16] and [23]. The next theorem is the counterpart of [16, Theorem 2.9], [12, Theorem 2.13] and [23, Theorem 3.14].

4.9. Theorem. Let $P$ be any $z_{l c}$-ideal in $L_{c c}(X)$. Then the following statements are equivalent. 
(1) $P$ is a prime ideal in $L_{c c}(X)$.

(2) $P$ contains a prime ideal in $L_{c c}(X)$.

(3) For all $f, g \in L_{c c}(X)$, if $f g=0$, then $f \in P$ or $g \in P$.

(4) For every $f \in L_{c c}(X)$, there is a zero-set in $Z_{l c}[P]$ on which $f$ does not change sign.

4.10. Corollary. Every prime ideal in $L_{c c}(X)$ is contained in a unique maximal ideal in $L_{c c}(X)$.

The following lemma is the counterpart of [12, Lemma 3.1] and if the proof of this lemma is applied verbatim we have a simple proof for the following lemma.

4.11. Lemma. Let $f, g, l \in L_{c c}(X), Z(f) \supseteq Z(g) \cap Z(l)$ and define

and

$$
h(x)=\left\{\begin{array}{rl}
0 & x \in Z(g) \cap Z(l) \\
\frac{f g^{2}}{g^{2}+l^{2}} & x \notin Z(g) \cap Z(l)
\end{array}\right.
$$

Then the following conditions hold.

$$
k(x)=\left\{\begin{array}{rl}
0 & x \in Z(g) \cap Z(l) \\
\frac{f l^{2}}{g^{2}+l^{2}} & x \notin Z(g) \cap Z(l)
\end{array}\right.
$$

(1) $|h| \vee|k|=|f|$.

(2) $f=h+k$.

(3) $f l^{2}=k\left(g^{2}+l^{2}\right)$ and $f g^{2}=h\left(g^{2}+l^{2}\right)$.

(4) $h, k \in L_{c c}(X)$.

(5) $C_{h} \supseteq C_{f} \cap C_{g} \cap C_{l}$ and $C_{k} \supseteq C_{f} \cap C_{g} \cap C_{l}$.

In view of the previous lemma, we may record some interesting facts, which follow. If $I$ and $J$ are two $z_{l c^{-}}$-ideals in $L_{c c}(X)$, then either $I+J=L_{c c}(X)$ or $I+J$ is a $z_{l c^{-}}$ ideal. More generally, if $A=\left\{A_{i}\right\}_{i \in I}$ is a collection of $z_{l c}$-ideals in $L_{c c}(X)$, then either $\sum_{i \in I} A_{i}=L_{c c}(X)$ or $\sum_{i \in I} A_{i}$ is a $z_{l c}$-ideal. It is well-known that each minimal prime ideal in a subring of the ring $R$ is the contraction of some minimal prime ideal of the ring $R$, see [19, p. 41], also minimal prime ideals of $C(X)$ (resp., $C_{c}(X)$ ) are $z$-ideals (resp., $z_{c}$-ideal), see [16] (resp., [12]). The next result is the counterpart of the latter facts for $L_{c c}(X)$.

4.12. Corollary. Every minimal prime ideal in $L_{c c}(X)$ is a $z_{l c}$-ideal.

The following result is a consequence of the two previous corollaries and Theorem 4.9.

4.13. Corollary. Let $\left\{P_{i}\right\}_{i \in I}$ be a collection of minimal prime ideals in $L_{c c}(X)$, then either $\sum_{i \in I} P_{i}=L_{c c}(X)$ or $P=\sum_{i \in I} P_{i}$ is a prime ideal in $L_{c c}(X)$.

Let us recall that an ideal $I$ of $C(X)$ is called absolutely convex if $f \in C(X), g \in I$ and $|f| \leqslant|g|$ implies that $f \in I$. The proofs of the following results can be obtained mutatis mutandis from the proofs of the corresponding results in [12] and [23].

4.14. Proposition. A prime ideal $P$ in $L_{c c}(X)$ is absolutely convex.

4.15. Proposition. The sum of a collection of semiprime ideals in $L_{c c}(X)$ is a semiprime ideal or is the entire ring $L_{c c}(X)$.

4.16. Proposition. Let $P$ be a prime ideal in $L_{c c}(X)$, then the ring $L_{c c}(X) / P$ is totally ordered and its prime ideals are comparable.

4.17. Proposition. Let $\left\{P_{i}\right\}_{i \in I}$ be a collection of semiprime ideals in $L_{c c}(X)$ such that at least one of $P_{i}$ 's is prime, then $\sum_{i \in I} P_{i}$ is a prime ideal or all of $L_{c c}(X)$.

4.18. Theorem. Let $I$ be an ideal in $L_{c c}(X)$, then $I$ and $\sqrt{I}$ have the same largest $z_{l c}$-ideal. 


\section{Co-locally countable completely regular spaces}

5.1. Definition. A Hausdorff space $X$ is called co-locally countable completely regular (briefly, $l c c$-completely regular) if whenever $F \subseteq X$ is a closed set and $x \in X \backslash F$, then there exists $f \in L_{c c}(X)$ with $f(F)=0$ and $f(x)=1$.

Clearly, a Hausdorff space $X$ is $l c c$-completely regular if and only if whenever $F \subseteq X$ is closed set and $x \in X \backslash F$, then $x$ and $F$ have two disjoint zero-set neighborhoods in $Z_{l c}(X)$. Consequently, there exist $g, h \in L_{c c}(X)$ with $x \in X \backslash Z(g) \subseteq Z(h) \subseteq X \backslash F$. In addition, $X$ is $l c c$-completely regular if and only if $F=\left\{Z(f): f \in L_{c c}(X)\right\}$ is a base for the closed sets in $X$ or equivalently if and only if $B=\left\{i n t Z(f): f \in L_{c c}(X)\right\}$ is a base for the open sets in $X$. The next proposition is the counterpart of [12, Proposition 4.3], [16, 3.11(a)] and [23, Proposition 4.3].

5.2. Proposition. Let $X$ be a lcc-completely regular space and $A, B$ be two disjoint closed sets in $X$ such that $A$ is compact, then there is $f \in L_{c c}(X)$ with $f(A)=0$ and $f(B)=1$.

5.3. Proposition. Let $X$ be a compact space. Then $X$ is lcc-completely regular if and only if $L_{c c}(X)$ separates points in $X$.

Recall that a space $X$ is said to be zero-dimensional if $X$ is a $T_{1}$ space with a base consisting of clopen sets. A Hausdorff space $X$ is called countably completely regular (briefly, c-completely regular) if whenever $F \subseteq X$ is a closed set and $x \notin F$, then there exists $f \in C_{c}(X)$ with $f(F)=0$ and $f(x)=1$, see [12, Definition 4.1]. In Proposition 4.4 of [12], it is shown that a topological space $X$ is zero-dimensional if and only if $X$ is ccompletely regular space. Since $C_{c}(X)$ is a subring of $L_{c c}(X)$, if $X$ is a zero-dimensional space then $X$ is a $l c c$-completely regular space. Similar to [16, Theorem 3.6], the next theorem can now be proved.

5.4. Theorem. Let $X$ be a Hausdorff space. Then $X$ is a lcc-completely regular space if and only if its topology coincides with the weak topology induced by $L_{c c}(X)$.

Let us recall that a space $X$ is a $P$-space if and only if $C(X)$ is a regular ring, see [16, $4 J]$. Also a space $X$ is $C P$-space (resp., $L C P$ - space) if and only if $C_{c}(X)\left(\operatorname{resp} ., L_{c}(X)\right)$ is a regular ring. In view of the [23, Proposition 4.9], if $L_{c c}(X)$ is regular, then $C_{c}(X)$ is regular too. The next definition is now in order.

5.5. Definition. A space $X$ is called a co-locally countably $P$-space (briefly, $L C C P$ space) if $L_{c c}(X)$ is regular.

Clearly, any $P$-space is $L C C P$-space. If $X$ is a $L C P$-space and $f \in L_{c c}(X)$, by regularity of $L_{c}(X)$, there exists $h \in L_{c}(X)$ such that $f=f^{2} h$. Put $g=h^{2} f$, therefore $f=f^{2} g$ and it is evident that $Z(f) \subseteq Z(g)$ and $g(x)=(f(x))^{-1}$, where $x \notin Z(f)$. Clearly, $C_{f}=C_{g}$ and so $g \in L_{c c}(X)$. Thus we have the following proposition.

\subsection{Proposition. Every LCP-space is LCCP-space.}

We should emphasize that if the proofs of [12, Theorem 5.5] and [23, Theorem 4.12] are applied verbatim we have a simple proof for the next theorem.

5.7. Theorem. A space $X$ is a LCCP-space if and only if every zero-set in $Z_{l c}(X)$ is open. Moreover, in this case whenever $\left\{f_{i}\right\}_{i \in \mathbb{N}}$ is a countable set in $L_{c c}(X)$, then $\bigcap_{i \in \mathbb{N}} Z\left(f_{i}\right)$ is an open zero-set in $Z_{l c}(X)$.

We recall that $X$ is a $P$-space if and only if every $G_{\delta}$-set is open, see $[16,4 \mathrm{~J}(3)]$. The following theorem is the counterpart of this fact for the $L C C P$-spaces. 
5.8. Theorem. Let $X$ be a lcc-completely regular LCCP-space. Then every $G_{\delta}$-set $A$ containing a compact set $S$, contains a zero-set in $Z_{l c}(X)$, which contain $S$. In particular, every lcc-completely regular LCCP-space is a P-space.

Let $M_{p}^{l c}=M_{p} \cap L_{c c}(X)$ and $O_{p}^{l c}=O_{p} \cap L_{c c}(X)$, where $p \in X, O_{p}$ is the ideal of $C(X)$ consisting of $f$ in $C(X)$ for which $Z(f)$ is a neighborhood of $p$ and $M_{p}$ is the maximal ideal of $C(X)$ consisting of all functions in $C(X)$ that vanish on $p$. Clearly, $M_{p}^{l c}$ is the kernel of the homomorphism $f \rightarrow f(p)$ of $L_{c c}(X)$ onto $\mathbb{R}$, in fact $\frac{L_{c c}(X)}{M_{p}^{l c}} \cong \mathbb{R}$ and hence $M_{p}^{l c}$ is a maximal ideal of $L_{c c}(X)$. Consequently, the Jacobson radical of $L_{c c}(X)$ is zero. The following theorem is similar to [16, 4J] and [12, Theorem 5.8].

5.9. Theorem. Let $X$ be a topological space. Then the following statements are equivalent.

(1) $X$ is a LCCP-space.

(2) $L_{c c}(X)$ is a regular ring.

(3) Each ideal in $L_{c c}(X)$ is a $z_{l c}$-ideal.

(4) Each prime ideal in $L_{c c}(X)$ is a maximal ideal.

(5) For each $p \in X, M_{p}^{l c}=O_{p}^{l c}$.

(6) Every zero-set in $Z_{l c}(X)$ is open.

(7) Each ideal in $L_{c c}(X)$ is an intersection of maximal ideals.

(8) For all $f, g \in L_{c c}(X),(f, g)=\left(f^{2}+g^{2}\right)$.

(9) For every $f \in L_{c c}(X), X \backslash Z(f)$ is $C$-embedded.

(10) If $\left\{f_{n}: n \in \mathbb{N}\right\} \subseteq L_{c c}(X)$, then $\bigcap_{n \in \mathbb{N}} Z\left(f_{n}\right)$ is an open zero-set in $Z_{l c}(X)$.

The next theorem is the counterpart of [13, Theorem 3.8] and [16, Theorem 4.11].

5.10. Theorem. Let $X$ be a lcc-completely regular space, then the following statements are equivalent.

(1) $X$ is compact.

(2) Every ideal of $L_{c c}(X)$ is fixed.

(3) Every maximal ideal of $L_{c c}(X)$ is fixed.

(4) Every prime ideal of $L_{c c}(X)$ is fixed.

5.11. Definition. A maximal ideal $M$ in $L_{c c}(X)$ is called a real maximal ideal of $L_{c c}(X)$ if $\frac{L_{c c}(X)}{M} \cong \mathbb{R}$. A topological space $X$ is called co-locally countably realcompact space (briefly, $l c c$-realcompact) if every real maximal ideal $M$ of $L_{c c}(X)$ is of the form $M=M_{p}^{l c}$ for some $p \in X$.

Let us we note that if $X$ is a compact zero-dimensional space, the corresponding $p \rightarrow M_{p}^{l c}$ is one-to-one from $X$ onto the set of maximal ideals of $L_{c c}(X), \operatorname{Max}\left(L_{c c}(X)\right)$ say, and therefore the space $X$ is homeomorphic to $\operatorname{Max}\left(L_{c c}(X)\right)$ with the Stone topology. The proof of the following fact which is similar to the its counterpart in [16, Theorem 8.3], is omitted.

5.12. Proposition. Two zero-dimensional lcc-realcompact spaces $X$ and $Y$ are homeomorphic if and only if $L_{c c}(X) \cong L_{c c}(Y)$.

The counterparts of the next theorem are already proved for the rings $C(X), C_{c}(X)$, and $L_{c}(X)$, in [16], [13], and [23], respectively. We present its proof for the sake of completeness.

5.13. Theorem. Let $X$ and $Y$ be two lcc-completely regular compact spaces. Then $X$ and $Y$ are homeomorphic if and only if $L_{c c}(X) \cong L_{c c}(Y)$. In particular, if $X$ and $Y$ are compact zero-dimensional spaces, then $L_{c c}(X) \cong L_{c c}(Y)$ if and only if $C_{c}(X) \cong C_{c}(Y)$. 
Proof. Obviously if $L_{c c}(X) \cong L_{c c}(Y)$, then $\operatorname{Max}\left(L_{c c}(X)\right) \cong \operatorname{Max}\left(L_{c c}(Y)\right)$ with the Stone topology and hence $X$ and $Y$ are homeomorphic. Conversely, if $\varphi: X \rightarrow Y$ is a homeomorphism from $X$ onto $Y$ and $f \in L_{c c}(Y)$, then we claim that $f o \varphi \in L_{c c}(X)$. Since $f \in L_{c c}(Y)$, we infer that $C_{f}=\bigcup_{i \in I} V_{i}$ is cocountable, where for each $i \in I, V_{i}$ is open in $Y$ with $\left|f\left(V_{i}\right)\right| \leqslant \aleph_{0}$. Put $U_{i}=\varphi^{-1}\left(V_{i}\right)$, where $i \in I$, clearly $U_{i}$ is an open set in $X$ and $\left|f o \varphi\left(U_{i}\right)\right|=\left|f o \varphi\left(\varphi^{-1}\left(V_{i}\right)\right)\right|=\left|f\left(V_{i}\right)\right| \leqslant \aleph_{0}$, thus $\bigcup_{i \in I} U_{i} \subseteq C_{f o \varphi}$. Now we have $\bigcup_{i \in I} U_{i}=\bigcup_{i \in I} \varphi^{-1}\left(V_{i}\right)=\varphi^{-1}\left(\bigcup_{i \in I} V_{i}\right)$. As $\bigcup_{i \in I} V_{i}$ is cocountable, $\bigcup_{i \in I} U_{i}$ is cocountable, too. This completes the proof.

We recall that a commutative ring $R$ is said to be selfinjective (resp., $\aleph_{0}$-selfinjective) if every homomorphism $f: I \rightarrow R$, where $I$ is an ideal (resp., a countably generated ideal) in $R$, can be extended to an homomorphism $\hat{f}: R \rightarrow R$. Let us also recall that a subset $S$ of a commutative ring $R$ is said to be orthogonal, provided $x y=0$ for all $x, y \in S$ with $x \neq y$. If $R$ is a commutative ring and $S, T$ are two disjoint subsets in $R$ with $S \cup T$ an orthogonal set in $R$, then $r \in R$ is said to separate $S$ from $T$, if $r s^{2}=s$ for all $s \in S$ and $r \in A n n(T)$. We also recall that if $R$ is a reduced ring, then $R$ is selfinjective (resp., $\aleph_{0}$-selfinjective) if and only if $R$ is regular and whenever $S \cup T$ is an orthogonal (countable orthogonal) set in $R$ with $S \cap T=\emptyset$, then there is an element in $R$ which separates $S$ from $T$. If the proof of [12, Lemma 6.7] is applied verbatim, we have a simple proof for the following lemma.

5.14. Lemma. The following statements are equivalent in $L_{c c}(X)$.

(1) If $S \cup T$ is an orthogonal set in $L_{c c}(X)$ with $S \cap T=\emptyset$, then there exists $f \in L_{c c}(X)$ which separates $S$ from $T$.

(2) For any orthogonal set $L$ in $L_{c c}(X)$ there exists $f \in L_{c c}(X)$ with $g^{2} f=g$ for all $g \in L$.

It is also worth mentioning that commutative regular rings are rarely $\aleph_{0}$-selfinjective, see $[17,14.7]$. But fortunately, similarly to the rings $C(X), C_{c}(X)$ and $L_{c}(X)$, regularity and $\aleph_{0}$-selfinjectivity of $L_{c c}(X)$ coincide, see [12], [23]. In [12] and [23] for showing the latter fact, they invoke [21, Lemma 1.9], but we prove its counterpart for $L_{c c}(X)$, without appealing to this lemma.

5.15. Theorem. Let $X$ be a topological space $X$. Then $L_{c c}(X)$ is regular if and only if $L_{c c}(X)$ is $\aleph_{0}$-selfinjective.

Proof. If $L_{c c}(X)$ is $\aleph_{0}$-selfinjective, then $L_{c c}(X)$ is regular by $[12$, Lemmas $6.7,6.8$, Remark 6.9]. Conversely, by the aforementioned facts we must show that if $S=\left\{f_{n}\right\}_{n \in \mathbb{N}}$ is an orthogonal subset in $L_{c c}(X)$, then there exists $f \in L_{c c}(X)$ such that $f f_{n}^{2}=f_{n}$ for each $f_{n} \in S$. Since $L_{c c}(X)$ is a regular ring, we infer that $\bigcap_{n \in \mathbb{N}} Z\left(f_{n}\right)=Z(h)$ is an open zero-set in $L_{c c}(X)$, by Theorem 5.9. Suppose that $G_{n}=X \backslash Z\left(f_{n}\right)$ for each $n \in \mathbb{N}$. For each $i \neq j$ since $f_{i} f_{j}=0$, we have $G_{i} \cap G_{j}=\emptyset$. If we define $G=\bigcup_{i \in \mathbb{N}} G_{n}$, then $X=\left(\bigcup_{n \in \mathbb{N}} G_{n}\right) \cup X \backslash G$. Therefore, we may define $f: X \rightarrow \mathbb{R}$ by $f(x)=\left(f_{n}(x)\right)^{-1}$ if $x \in G_{n}$ and $f(x)=0$, otherwise. The function $f$ is continuous by [16, 1A(2)] and clearly $f_{n}^{2} f=f_{n}$, for all $n \in \mathbb{N}$. It remains to be shown that $f \in L_{c c}(X)$, to do this we claim that $\bigcap_{n \in \mathbb{N}} C_{f_{n}} \subseteq C_{f}$. Assume that $x \in \bigcap_{n \in \mathbb{N}} C_{f_{n}}$, if $x \in X \backslash G$, then $f(X \backslash G)=0$ and since $X \backslash G$ is an open set in $X$, we conclude that $x \in C_{f}$. If $x \in G_{n}$, for some $n \in \mathbb{N}$, then there exists an open set $U_{x}$ containing $x$ with $\left|f_{n}\left(U_{x}\right)\right| \leqslant \aleph_{0}$. Hence, $U_{x} \cap G_{n}$ is an open set in $X$ contains $x$ and $\left|f\left(U_{x} \cap G_{n}\right)\right|=\left|f_{n}\left(U_{x} \cap G_{n}\right)\right| \leqslant\left|f_{n}\left(U_{x}\right)\right| \leqslant \aleph_{0}$, i.e., $x \in C_{f}$. Consequently, we have $\bigcap_{n \in \mathbb{N}} C_{f_{n}} \subseteq C_{f}$ and since $f_{n} \in L_{c c}(X)$ for all $n \in \mathbb{N}$, we infer that $f \in L_{c c}(X)$. 


\section{The socle of $L_{c c}(X)$}

Let us recall that for a commutative ring $R$ the socle of $R$, denoted by $\operatorname{Soc}(R)$, is the intersection of all essential ideals of $R$ which is also equal to the sum of all minimal ideals of $R$, see [25]. Karamzadeh and Rostami in [24] have shown that the socle of $C(X)$ is equal to all functions which vanish everywhere except on a finite number of points of $X$, that is $\operatorname{Soc}(C(X))=C_{F}(X)$. We also recall that if $I$ is a minimal ideal of $C(X)$, then $I=e C(X)$, where $e$ is an idempotent such that $e(x)=1$ and $Z(e)=X \backslash\{x\}$, where $x$ is an isolated point of $X$, see [24, Proposition 3.1], in this case one can easily see that $I=e L_{c c}(X)$ (note, although this is a consequence of Brauer's Lemma in noncommutative ring theory, but it can also be obtained from the fact, that in any commutative ring $R$, a finitely generated idempotent ideal is generated by an idempotent). Similarly to the rings $C(X), C_{c}(X)$ and $L_{c}(X)$ the Peirce decomposition $L_{c c}(X)=e L_{c c}(X) \oplus(1-e) L_{c c}(X)$ holds. Since $(1-e) L_{c c}(X)=(1-e) C(X) \cap L_{c c}(X)$ is a maximal ideal in $L_{c c}(X)$, we infer that $I=e L_{c c}(X)$ is a minimal ideal in $L_{c c}(X)$. Hence, every minimal ideal of $C(X)$ is a minimal ideal in $L_{c c}(X)$, too. Consequently, $C_{F}(X)$ is an ideal in $L_{c c}(X)$ and $C_{F}(X) \subseteq \operatorname{Soc}\left(L_{c c}(X)\right)$. It is also worth mentioning that since $\operatorname{Soc}\left(L_{c c}(X)\right)$ is a semisimple $L_{c c}(X)$-module, we infer that $C_{F}(X)$ is a direct summand of $\operatorname{Soc}\left(L_{c c}(X)\right)$ as a $L_{c c}(X)$-module. Furthermore, recall that in any commutative ring $R$, if $I$ is a finitely generated ideal and $I=I^{2}$, then $I$ is generated by an idempotent, see [10]. In view of this fact, if $I$ is a minimal ideal in a reduced ring $R$, then $I=e R$, where $e$ is an idempotent element. We recall that if $Y$ is a subset of a space $X$, then the set of all $f \in C(X)$ such that $\left.f\right|_{Y}$ is constant is a subalgebra of $C(X)$, denoted by $C_{1}(Y)$. Also, we say that $Y$ is constant with respect to a subring $A$ of $C(X)$ if $A \subseteq C_{1}(Y)$, see [23, Definition 2.23]. Since the proofs of most of the results in this section are very similar to the proofs of their corresponding results in [23], we state them without proofs. The following lemma characterizes the minimal ideals of $L_{c c}(X)$.

6.1. Lemma. Let $I$ be a nonzero minimal ideal in $L_{c c}(X)$, then $I=e L_{c c}(X)$ where $e$ is an idempotent in $L_{c c}(X)$ such that $Z(1-e)$ is connected. Conversely, if $I=e L_{c c}(X)$ where $e \neq 0$ is an idempotent in $L_{c c}(X)$ such that $Z(1-e)$ is a constant subset of $X$ with respect to $L_{c c}(X)$, then $I$ is a minimal ideal in $L_{c c}(X)$.

Proof. Let $I$ be a nonzero minimal ideal in $L_{c c}(X)$, since $L_{c c}(X)$ is a reduced ring, we infer that $I=e L_{c c}(X)$, where $e$ is an idempotent element in $L_{c c}(X)$. If $Z(1-$ $e)$ is not connected, then there exists a nonempty clopen subset $A \subsetneq Z(1-e)$. The idempotent function $e_{1}$ which $A=Z\left(1-e_{1}\right)$ lies in $L_{c c}(X)$ and $A=Z\left(1-e_{1}\right) \subsetneq Z(1-e)$. Consequently, $e_{1}=e_{1} e$ but $e \neq e_{1} e$, hence $e_{1} L_{c c}(X) \subsetneq e L_{c c}(X)=I$ and this contradicts the minimality of $I$. Conversely, let $I=e L_{c c}(X)$, where $e \in L_{c c}(X)$ such that $Z(1-e)$ is a constant subset of $X$ with respect to $L_{c c}(X)$. Now, we define $\varphi: L_{c c}(X) \longrightarrow \mathbb{R}$ by $\varphi(f)=f(Z(1-e))$. Clearly $\operatorname{ker} \varphi=(1-e) L_{c c}(X)$ and $\frac{L_{c c}(X)}{(1-e) L_{c c}(X)} \cong \mathbb{R}$, therefore $(1-e) L_{c c}(X)$ is maximal ideal in $L_{c c}(X)$ and $I$ is minimal in $L_{c c}(X)$.

Similarly to [13, Proposition 5.3] and [23, Proposition 5.3], the next proposition characterizes the socle of $L_{c c}(X)$ topologically. We note that the following proposition is evident by [23, Remark 5.3].

6.2. Proposition. Let $f \in L_{c c}(X)$ be a nonunit element. If $f \in \operatorname{Soc}\left(L_{c c}(X)\right)$, then $X \backslash Z(f) \subseteq \bigcup_{n=1}^{k} A_{n}$ where $k \in \mathbb{N}$ and $\left\{A_{1}, A_{2}, \ldots, A_{k}\right\}$ is a set of mutually disjoint clopen connected subsets of $X$. Conversely, if $X \backslash Z(f) \subseteq \bigcup_{n=1}^{k} A_{n}$, where $k \in \mathbb{N}$ and $\left\{A_{1}, A_{2}, \ldots, A_{k}\right\}$ is a set of mutually disjoint clopen of constant subsets of $X$ with respect to $L_{c c}(X)$, then $f \in L_{c c}(X)$. In particular, $\operatorname{Soc}\left(L_{c c}(X)\right)$ is a $z_{l c}$-ideal in $L_{c c}(X)$. 
We note that the clopen subsets of a space $X$ which are constant with respect to $L_{c c}(X)$ may not be constant with respect to $L_{c}(X)$. Let $X=[0,1]$ with the usual topology, then the Cantor function $f$ is a nonconstant function that lies in $L_{c}(X)$, see [23, Remark 2.9]. As $X$ is a second countable space, $L_{c c}(X)=C_{c}(X)$ by Proposition 3.18 and hence the Cantor function is not in $L_{c c}(X)$. Hence, $X$ is a clopen set which is constant with respect to $L_{c c}(X)$ but it is not constant with respect to $L_{c}(X)$. The next fact characterizes spaces $X$ such that the socles of $L_{c c}(X)$ and $C_{c}(X)$ coincide.

6.3. Theorem. $\operatorname{Soc}\left(L_{c c}(X)\right)=\operatorname{Soc}\left(C_{c}(X)\right)$ if and only if the clopen connected subsets of $X$ coincide with the clopen constant subsets of $X$ with respect to $L_{c c}(X)$.

Proof. Let $\operatorname{Soc}\left(L_{c c}(X)\right)=\operatorname{Soc}\left(C_{c}(X)\right)$ and $A$ be a nonempty clopen constant subspace of $X$ with respect to $L_{c c}(X)$. We are to show that $A$ is connected. Clearly, there exists an idempotent function $e$ in $L_{c c}(X)$ with $A=Z(1-e)$ and by Lemma 6.1, $e \in$ $\operatorname{Soc}\left(L_{c c}(X)\right)=\operatorname{Soc}\left(C_{c}(X)\right)$. Hence, $A=Z(1-e)$ must be connected, by [13, Lemma 5.3]. Conversely, if $I$ is a minimal ideal in $L_{c c}(X)$, then $I=e L_{c c}(X)$ where $e$ is an idempotent such that $Z(1-e)$ is connected, by Lemma 6.1. Hence $I$ is a minimal ideal in $C_{c}(X)$, by [13, Lemma 5.1]. Now, let $I$ be a nonzero minimal ideal in $C_{c}(X)$, so $I=e C_{c}(X)$, where $e$ is an idempotent and $Z(1-e)$ is a clopen connected subset in $X$, by [13, Lemma 5.1]. Since clopen connected subsets of $X$ coincide with the clopen constant subsets of $X$ with respect to $L_{c c}(X)$, we infer that $I=e L_{c c}(X)$ is a minimal ideal of $L_{c c}(X)$ by Lemma 6.1 .

It is clear that the socle of $C(X)$ and the socle of $C_{c}(X)$ coincide if and only if every proper nonempty clopen connected subspace of $X$ is singleton (i.e., consisting of an isolated point), see [12, Theorem 5.6]. Also in [23] the spaces $X$ for which $\operatorname{Soc}\left(L_{c}(X)\right)=C_{F}(X)$ is studied and in the following theorem we determines spaces $X$ that $\operatorname{Soc}\left(L_{c c}(X)\right)=C_{F}(X)$. The proof of this theorem is similar to Theorem 5.5 in [23] and we omit it.

6.4. Theorem. If every proper nonempty clopen connected subset of $X$ is singleton, (e.g., any totally disconnected space), then $\operatorname{Soc}\left(L_{c c}(X)\right)=C_{F}(X)$. Conversely, if $\operatorname{Soc}\left(L_{c c}(X)\right)$ $=C_{F}(X)$, then every proper nonempty clopen constant subspace of $X$ with respect to $L_{c c}(X)$ is singleton.

The next corollary is a consequence of Theorem 6.3 and Theorem 6.4.

6.5. Corollary. $\operatorname{Soc}\left(L_{c c}(X)\right)=C_{F}(X)=\operatorname{Soc}\left(C_{c}(X)\right)$ if and only if each clopen connected subset of $X$ consists of a single isolated point. Thus, if $X$ is zero-dimensional or totally disconnected, then $\operatorname{Soc}\left(L_{c c}(X)\right)=C_{F}(X)=\operatorname{Soc}\left(C_{c}(X)\right)$.

We recall that an ideal in a commutative ring $R$ is essential if it intersects every nonzero ideal of $\mathrm{R}$ nontrivially. The following corollary is similar to [13, Corollary 5.4] and [23, Corollary 5.7]. We should emphasize that if these proofs are applied verbatim we have a simple proof for the next corollary, too.

6.6. Corollary. Let $X$ be a lcc-completely regular space, and $\operatorname{Soc}\left(L_{c c}(X)\right)=\sum_{i \in I} \bigoplus$ $e_{i} L_{c c}(X)$, where $e_{i} L_{c c}(X)$ is a nonzero minimal ideal of $L_{c c}(X)$, and $e_{i}$ is an idempotent for each $i \in I$. Put $Y=\bigcup_{i \in I} Z\left(1-e_{i}\right)$, then $\operatorname{Soc}\left(L_{c c}(X)\right)$ is essential in $L_{c c}(X)$ if and only if $Y$ is dense in $X$.

It is well-known that $C_{F}(X)$ is never a prime ideal of $C(X)$, see [10, Proposition 1.2], also $C_{F}(X) \neq(0)$ if and only if $X$ has an isolated point. Similarly to [23, Proposition 5.8], the next proposition characterizes spaces $X$ such that $C_{F}(X)$ is a prime ideal in $L_{c c}(X)$. 
6.7. Proposition. Let $|I(X)|<\infty$, where $I(X)$ is the set of isolated points in $X$. If $C_{F}(X) \neq(0)$ is a prime ideal in $L_{c c}(X)$, then $X \backslash I(X)$ is connected in $X$. Conversely, if $X \backslash I(X)$ is constant with respect to $L_{c c}(X)$, then $C_{F}(X) \neq(0)$ is prime in $L_{c c}(X)$.

In the following corollary, we consider spaces $X$ such that $C_{F}(X)$ is not a prime ideal in $L_{c c}(X)$. If the proof of Corollary 5.9 of [23] is applied verbatim, we have a simple proof of the next fact, too.

6.8. Corollary. If $I(X)$ is an infinite set or $Y=X \backslash I(X)$ is disconnected, then $C_{F}(X)$ is never a prime ideal in $L_{c c}(X)$.

The following theorem shows that for the spaces $X$ in which there exist certain constant subsets with respect to $L_{c c}(X), L_{c c}(X)$ can not be isomorphic to any $C(Y)$.

6.9. Theorem. Let $|I(X)|<\infty$ and $Y=X \backslash I(X)$ be constant with respect to $L_{c c}(X)$ (note, in this case $L_{c c}(X)=C^{F}(X)$ ). Then there is no space $Y$ with $L_{c c}(X) \cong C(Y)$.

Proof. Let $I(X)$ be finite and $X \backslash I(X)$ is constant with respect to $L_{c c}(X)$, then by Proposition 6.7, $C_{F}(X)$ is a prime ideal in $L_{c c}(X)$. If there exists a space $Y$ such that $L_{c c}(X) \cong C(Y)$, then $\operatorname{Soc}\left(L_{c c}(X)\right) \cong C_{F}(Y)$. Since $\operatorname{Soc}\left(L_{c c}(X)\right)$ is a $z_{l c}$-ideal containing a prime ideal $C_{F}(X)$, by Theorem $4.9, \operatorname{Soc}\left(L_{c c}(X)\right)$ is a prime ideal in $L_{c c}(X)$. Therefore $C_{F}(Y)$ is a prime ideal in $C(Y)$, which is a contradiction.

It is worth mentioning that if we replace $L_{c c}(X)$ by $L_{c F}(X)$ or $L_{c 1}(X)$ in this section, then all results of this section remain valid for these two rings, too.

Acknowledgment. We are grateful to professor O. A. S. Karamzadeh for introducing the topic of this study to us and for his advice and encouragment during the preparation of this article. Also, the authors would like to thank the well-informed referee for reading the article carefully and giving very useful suggestions.

\section{References}

[1] Atiyah, M. F. and Macdonald, I. G. Introduction to commutative algebra, Addison-Wesley, Reading, Mass., 1969.

[2] Azarpanah, F. Intersection of essential ideals in $C(X)$, Proc. Amer. Math. Soc. 125, 2149$2154,1997$.

[3] Azarpanah, F. and Karamzadeh, O. A. S. Algebraic characterization of some disconnected spaces, Italian. J. Pure Appl. Math. 12, 155-168, 2002.

[4] Azarpanah, F., Karamzadeh, O. A. S. and Rahmati, S. $C(X)$ VS. $C(X)$ modulo its socle, Colloq. Math. 3, 315-336, 2008.

[5] Bhattacharjee, P., Knox, M. L. and Mcgovern, W. WM. The classical ring of quotients of $C_{c}(X)$, Appl. Ge Topol. 15, no. 2, 147-154, 2014.

[6] Azarpanah, F., Manshoor, F. and Mohamadian, R. Connectedness and compactness in $C(X)$ with the $m$-topology and generalized $m$-topology, Topology and its applications 159, 3486-3493, 2012.

[7] Dovgoshey, O., Martio, O., Ryazanov, V. and Vuopinen, M. The Cantor function, Expo. Math. 24, 1-37, 2006.

[8] Dube, T. Contracting the socle in rings of continuous functions, Rend. Semin. Mat. Univ. Padova 123, 37-53, 2010.

[9] Engelking, R. General topology, Heldermann Verlag Berlin, 1989.

[10] Estaji, A. A. and Karamzadeh, O. A. S. On $C(X)$ modulo its socle, Comm. Algebra 31, 1561-1571, 2003.

[11] Ganster, M. A note on strongly lindelöf spaces, Soochow J. Math. 15 (1), 99-104, 1989.

[12] Ghadermazi, M., Karamzadeh, O. A. S. and Namdari, M. On the functionally countable subalgebra of $C(X)$, Rend. Sem. Mat. Univ. Padova, 129, 47-69, 2013. 
[13] Ghadermazi, M., Karamzadeh, O. A. S. and Namdari, M. $C(X)$ versus its functionally countable subalgebra, submitted in 2013.

[14] Ghadermazi, M. and Namdari, M. On $\alpha$-scattered spaces, Far East J. Math. Sci.(FJMS), 32 (2), 267-274, 2009.

[15] Ghasemzadeh, S. G., Karamzadeh, O. A. S. and Namdari, M. The super socle of the ring of continuous functions, Mathematica Slovaka, to appear.

[16] Gillman, L. and Jerison, M., Rings of continuous functions, Springer-Verlag, 1976.

[17] Goodearl, K. R., Von Neumann Regular Rings, Pitman, 1979.

[18] Henriksen, M., Raphael, R. and Woods, R. G. SP-scattered spaces; a new generalization of scattered spaces, Comment. Math. univ. carolin 48, no. 3, 487-505, 2007.

[19] Kaplansky, I. Commutative rings, Boston: Allyn and Bacon, Inc, 1970.

[20] Karamzadeh, O. A. S. On a question on Matlis, Comm. Algebra, 25, 2717-2726, 1997.

[21] Karamzadeh, O. A. S. and Koochakpour, A. A. On $\aleph_{0}$-selfinjectivity of strongly regular rings, Comm. Algebra 27, 1501-1513, 1999.

[22] Karamzadeh, O. A. S. and Motamedi, M. A note on rings in which every maximal ideal is generated by a central idempotent, Proc. Japan Acad., 58 Ser. A, 124-125, 1982.

[23] Karamzadeh, O. A. S., Namdari, M. and Soltanpour, S. On the locally functionally countable subalgebra of $C(X)$, Appl. Gen. Topol., 16, no (2) 183-207, 2015.

[24] Karamzadeh, O. A. S. and Rostami, M. On the intrinsic topology and some related ideals of $C(X)$, Proc. Amer. Math. Soc. 93, 179-184, 1985.

[25] Lambek, J. Lectures on rings and modules, Blaisdell, 1966.

[26] Levy, R. and Rice, M.D. Normal P-spaces and the $G_{\delta}$-topology, Colloq. Math. 47, 227-240, 1981.

[27] Namdari, M. and Veisi, A. The subalgebra of $C_{c}(X)$ consisting of elements with countable image versus $C(X)$ with respect to their rings of quotients, Far East J. Math. Sci. (FJMS), 59, 201-212, 2011.

[28] Pelczynski, A. and Semadeni, Z. Spaces of continuous functions (III), Studia Math. 18, 211-222, 1959.

[29] Rudd, D. On two sum theorems for ideals of $C(X)$, Michigan Math. J. 17, 139-141, 1970.

[30] Rudin, W. Continuous functions on compact spaces without perfect subsets, Proc. Amer. Math. Soc. 8, 39-42, 1957.

[31] Rudin, M. E. and Rudin, W. Continuous functions that are locally constant on dense sets, J. Funct. Anal. 133, 120-137, 1995.

[32] Willard, S. General topology, Addison-Wesley, Reading, MA, 1970. 